\title{
Power quality measurement of MV distribution system after connection of small power plant Jakusevec
}

\author{
Mate Lasic ${ }^{1}$, Marko Sporec ${ }^{2}$, Irena Klaric ${ }^{2}$ \\ ${ }^{1}$ Končar Electrical Engineering Institute \\ Fallerovo setaliste 22, 10002 Zagreb (Croatia) \\ phone:+385 13667 337, fax.:+385 13667 309, e-mail: mate.lasic@koncar-institut.hr \\ ${ }^{2}$ HEP-Operator distribucijskog sustava d.o.o., DP Elektra Zagreb \\ Gunduliceva 32, 10000 Zagreb (Croatia) \\ phone:+385 1 4601111, fax.: :+385 1 4856329, e-mail: marko.sporec@hep.hr, irena.klaric@hep.hr
}

\begin{abstract}
Generation of electrical energy in large conventional power plants (thermal, hydro and nuclear) in last ten years worldwide is being supplemented by small generation utilities that are fuelled by renewable sources, waste etc.

In Republic of Croatia few years ago first small power plants projects were started. One such project was constructing small thermal power plant as a part of sanitary landfill treatment process in Zagreb, capital of Republic of Croatia. It is first power plant in Republic of Croatia of that kind which is fuelled by landfill gas that is result of decomposition process.

After the commissioning of small thermal power plant (STPP) Jakusevec a detailed analysis of voltages and currents at the point of connection to the local distribution network was conducted. Results of that analysis are given in this paper.
\end{abstract}

Key words: Distributed generation, distribution system, power quality, harmonics and flicker.

\section{Introduction}

STPP Jakusevec operates parallel with local distribution network, which is of rated voltage $10 \mathrm{kV}$ and consists of cables exclusively. It is specified that STPP delivers all produced energy in distribution network, and needs for own consumption are fulfilled from local low voltage distribution network.

Supply point for local distribution network is HV/MV $(110 / 10 \mathrm{kV})$ substation Sopot, with 2 power transformers of 40 MVA each. Medium voltage (MV) part of substation consists of 35 feeder cables that supply more than $200 \mathrm{MV} / \mathrm{LV}(10 / 0,4 \mathrm{kV})$ substations.

Local distribution network is ring network, that means feeder cable from one supply point supplies a certain number of MV/LV substations and it comes back to the starting supply point.

Considering the size of distribution network to which it is connected to, STPP with it's production of 2 MW does not represent a significant power source, so theoretically it's not to be expected that it's operation could have any significant effect on entire distribution network of that area, but only on the feeder it's connected to (fig. 1).

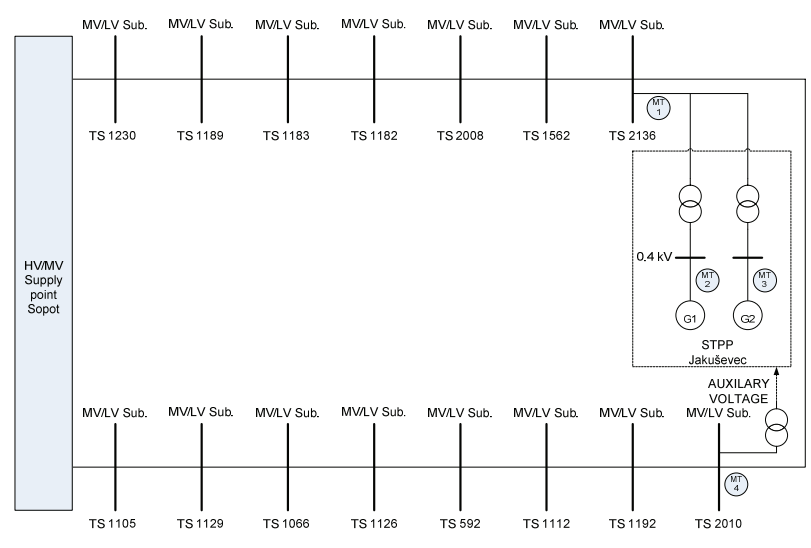

Fig. 1 Connection of the STPP Jakusevec to the distribution grid

Power generation utility has two generators of rated power $1168 \mathrm{~kW}$ each. Generators are connected to the MV distribution network over two power transformers of rated power $1250 \mathrm{kVA}$ each. Eventually, when the amount of landfill gas increases (in approximately 5 years) as the landfill volume reaches its maximum, third generator of the same characteristics will be installed.

Gas installation on the landfill site is made of gas wells connected into a gas pipes network that ends in a gas collecting station. Landfill gas is being collected with compressor pumps and from the collecting station is being conducted to the torches or generators. Torches are used to burn gas when generators are, for example, in maintenance. In that case, heat energy isn't exploited, but it's wasted in the atmosphere. Gas pipe network that delivers gas to the generators is approximately $8 \mathrm{~km}$ long. Landfill gas is made of 40-60 \% methane $\left(\mathrm{CH}_{4}\right), 20-35 \%$ carbon dioxide $\left(\mathrm{CO}_{2}\right)$ and $0-3 \%$ oxygen $\left(\mathrm{O}_{2}\right)$, and as such is excellent fuel for thermal power plants even at small amounts. 
Parallel operation of STPP Jakusevec with distribution network effects network's voltage conditions inevitable. In order to evaluate effects of that connection, it was necessary to perform measurements of power quality parameters at the point where electric energy is delivered in to the distribution network.

\section{Description of measurement}

Small thermal power plant Jakusevec is connected to MV electrical distribution network in MV/LV substation TS 2136. Point of common coupling (PCC) is placed in that substation on MV level (measurement point M1). In that point was power quality measured during two periods: from 2005-05-23 to 2005-05-30 and from 2005-05-31 to 2005-06-07.

At the same time RMS values of currents, voltages, active and reactive powers, voltage and current harmonics were made on both generators (on G1 in first measurement period - measurement point M2, and on G2 in second measurement period - measurement point M3). Measurements during switching operations were made on 2005-06-30 at the point of common coupling on MV side in TS 2136 (measurement point M1), on both generators on LV level (measurement points M2 and M3), and on the MV side in substation TS 2010 (measurement point M4).

Measurement points are marked on fig. 1. For measurements were following instruments used: Metrel's MI 2192, Lem Norma's Memobox 808 and Topas 1000, Dranetz BMI's PP4300 and PowerXplorer PX5.

Power quality measurement results analysis was conducted according to international standards and technical reports regarding power quality in distribution networks at medium voltage level [1], [2] and [3].

\section{Measurement results}

Power quality (PQ) measurements were made according to European standard EN 50160 [1] during two 7 day long periods. During the first period both generators were in operation, but in different modes (as shown on fig. 2). During second measurement period only G2 was in operation. Regardless of generators operating mode PQ at PCC was completely compliant with EN 50160, as show on fig. 3 and 4.

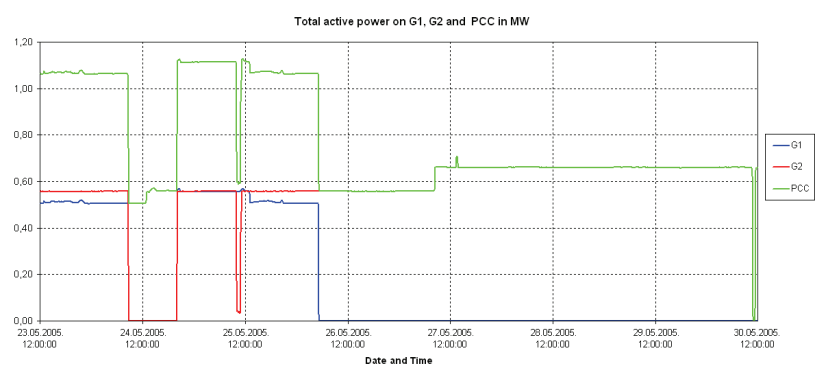

Fig. 2 Active power flow on G1 (M2), G2 (M3) and total at PCC (M1) during period from 2005-05-23 to 2005-05-30

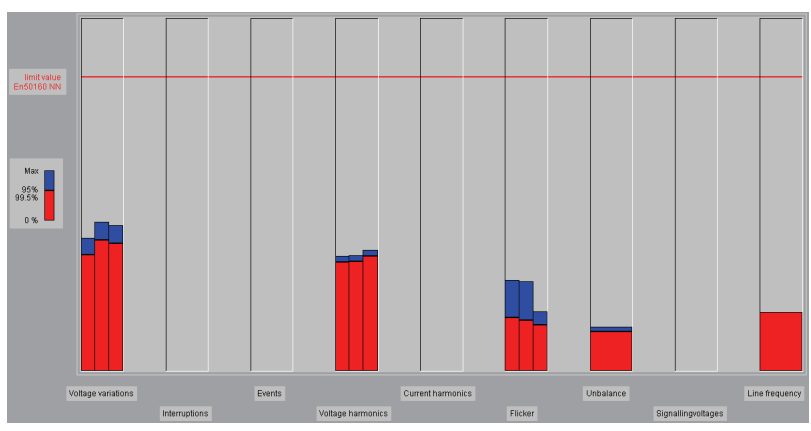

Fig. 3 Graphical overview of PQ data measured at PCC (M1) according to EN 50160 during period from 2005-05-23 to 2005-05-30

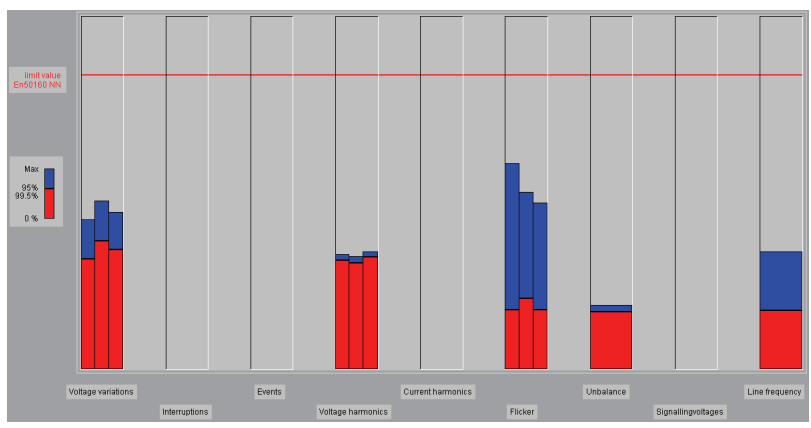

Fig. 4 Graphical overview of PQ data measured at PCC (M1) according to EN 50160 during period from 2005-05-31 to 2005-06-07

On fig. 5 comparison of phase currents and voltages at PCC (M1) during time period from 2005-05-23 to 200505-30 is given. From the figure it can be seen that changes in power flow from STPP to the network have no influence on voltage size at PCC.

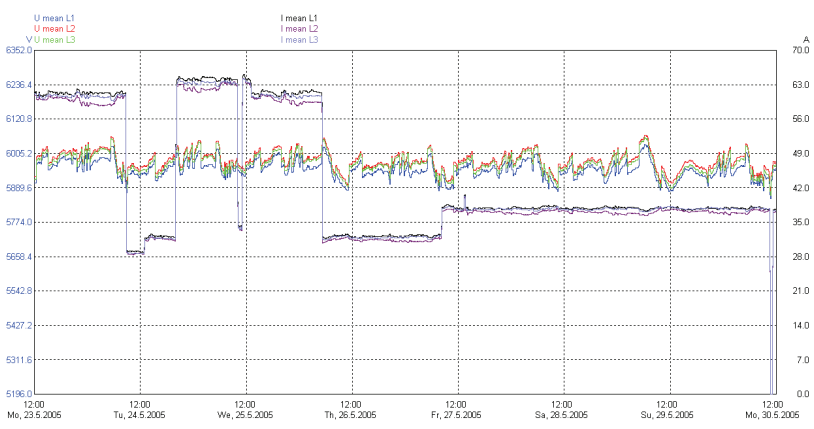

Fig. 5 Comparison of phase voltages and currents at PCC (M1) during time period from 2005-05-31 to 2005-06-07

Figure 6 shows short and long term flicker severity as well as possible causes for it $(10 \mathrm{~ms}$ minimum and maximum voltage values and current values at the moment voltage extreme was reached) in phase L1 at PCC (M1) during time period from 2005-05-23 to 2005.05.30. From this graph it is evident that voltage variations that cause flickers are not result of changes in generators load. 


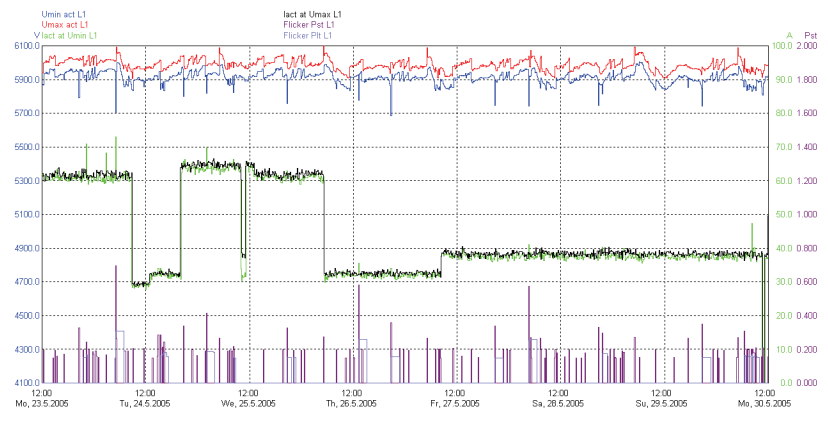

Fig. 6 Flicker measurement results analysis at PCC (M1) during time period from 2005-05-23 to 2005-05-30

On fig. 7 10-minute average values of fifth voltage and current harmonic (relatively to fundamental harmonic) as well as total active power on G2 (M3) are given during time period from 2005-05-31 to 2005-06-07. From the graph it is noticed that fifth current harmonic is result of network's voltage distortion.

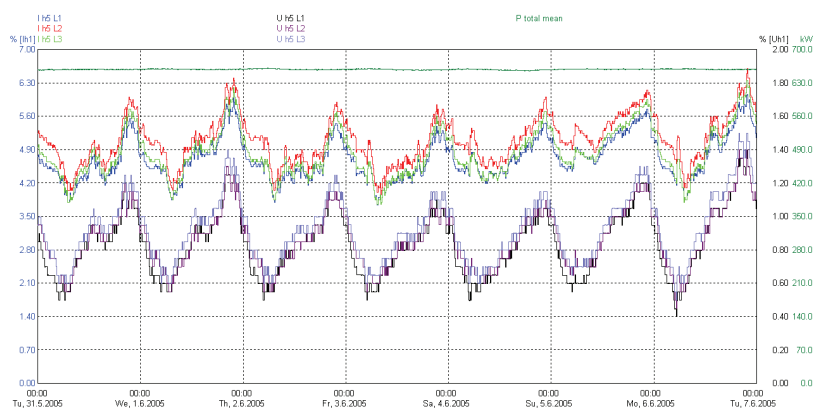

Fig. 7 Fifth harmonic current and voltage measurement analysis on G2 (M3) during time period from 2005-05-31 to 2005-06-07

Fig. 8 gives comparison of 10-minute average values of seventh voltage and current harmonic (relatively to fundamental harmonic) as well as total active power on G2 (M3) are given during time period from 2005-05-31 to 2005-06-07. From the graph it is noticed that seventh current harmonic is result of network's voltage distortion.

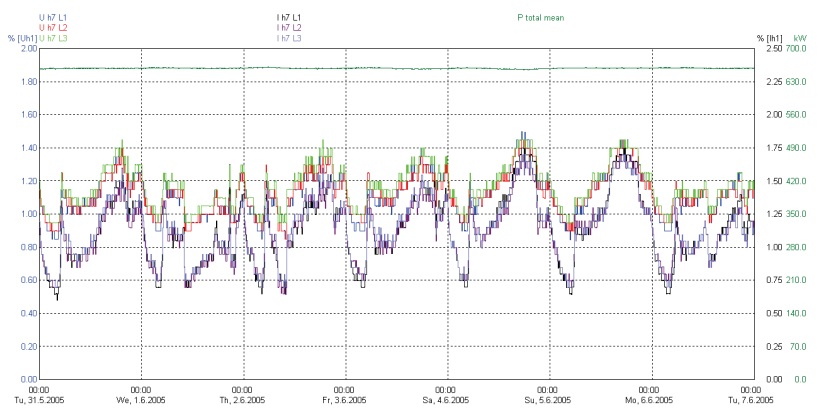

Fig. 8 Seventh harmonic current and voltage measurement analysis on G2 (M3) during time period from 2005-05-31 to 2005-06-07

On fig. 9 is given comparison of voltage total harmonic distortion (THD) for all three phases (10-min average) at PCC (M1) and generator G2 (M3) during time period from 2005-05-31 to 2005-06-07. From this graph is evident that voltage distortion is more present on $\mathrm{MV}$ side (at the PCC).

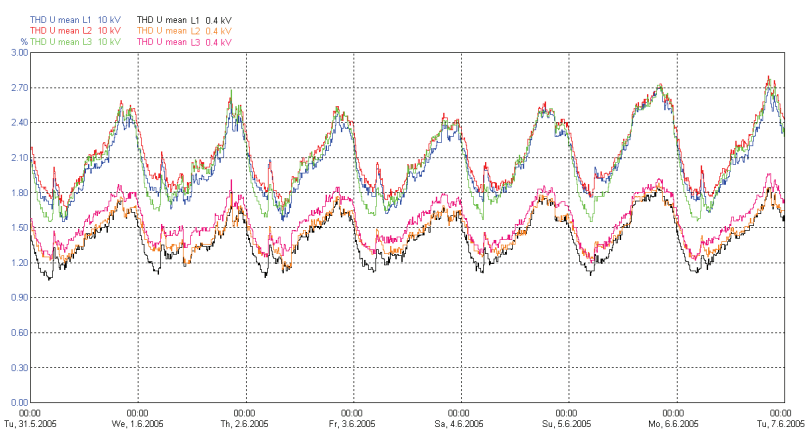

Fig. 9 Voltage THD comparison at PCC (M1) and on G2 (M3) during time period from 2005-05-31 to 2005-06-07

Fig. 10 shows oscillograms of currents and voltages at the moment of switching on generator G2 at 12:30 on 2005-06-30. From this figure it can be seen that switching on G2 does not cause any sharp changes of voltages or currents.

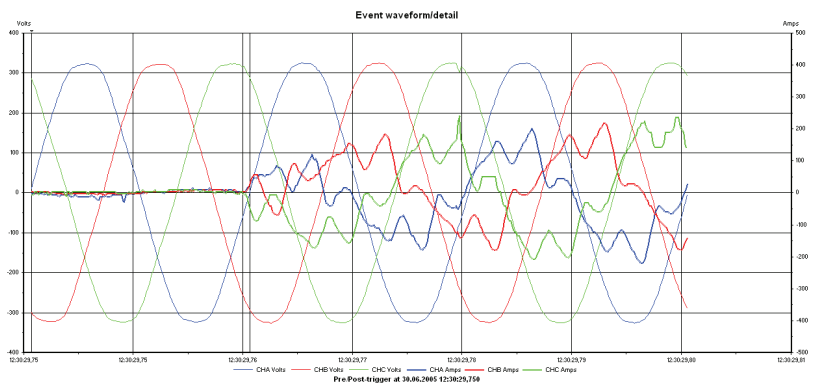

Fig. 10 Oscillogram of currents and voltages at the moment of switching on generator G2 on 2005-06-30

On fig. 12 oscillograms of currents and voltages at PCC (M1) are given at the moment of disconnecting a part of MV distribution network in TS 2010 at 11:27 on 200506-30. During the switching off both generators were in operation, with G1 giving $600 \mathrm{~kW}$ (technical minimum is $350 \mathrm{~kW}$ ), and G2 giving $660 \mathrm{~kW}$.

After the disconnection generators were left in island mode with only a part of MV distribution network connected that consisted of MV/LV substations TS 2136, TS 1562 and TS 2008 (fig. 11). Consumption of this part of the network was around $100 \mathrm{~kW}$, so generators started to speed up because load was too small. $200 \mathrm{~ms}$ after island mode was established due to overvoltage protection, generator switches were switched off and generators were stopped. On fig. 13 time diagram of voltages during the generators switch off is given. 


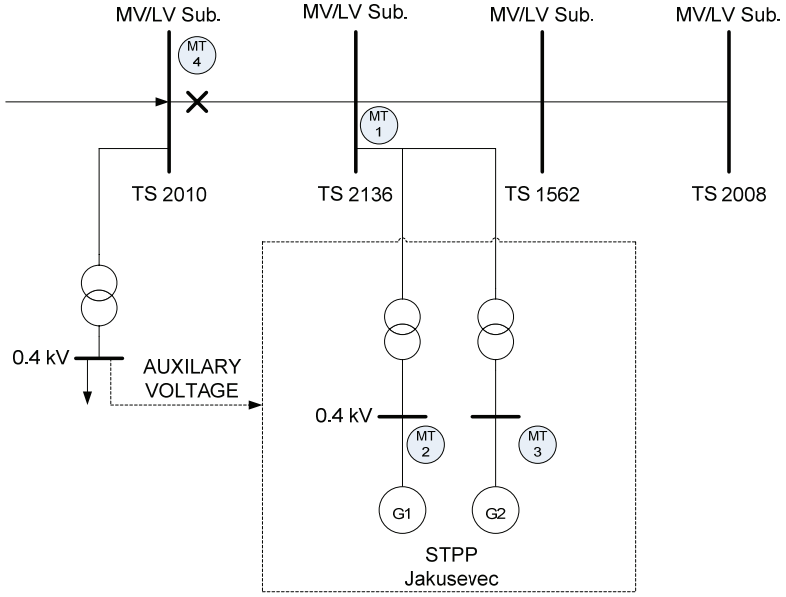

Fig. 11 Island operating mode of STPP Jakusevec

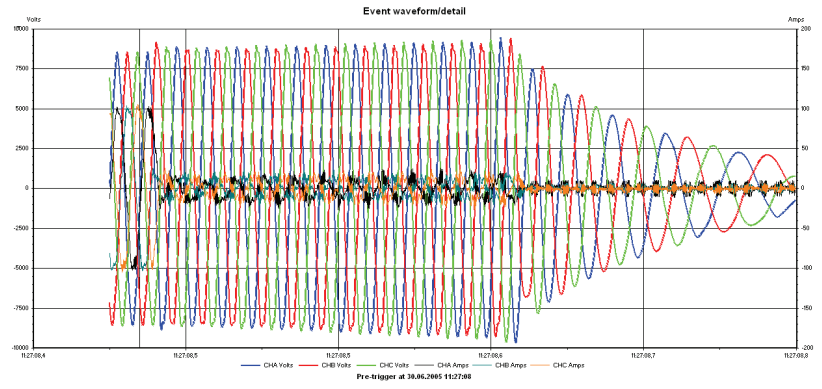

Fig. 12 Oscilogram of currents and voltages during island operating mode at the PCC (M1) on 2005-06-30

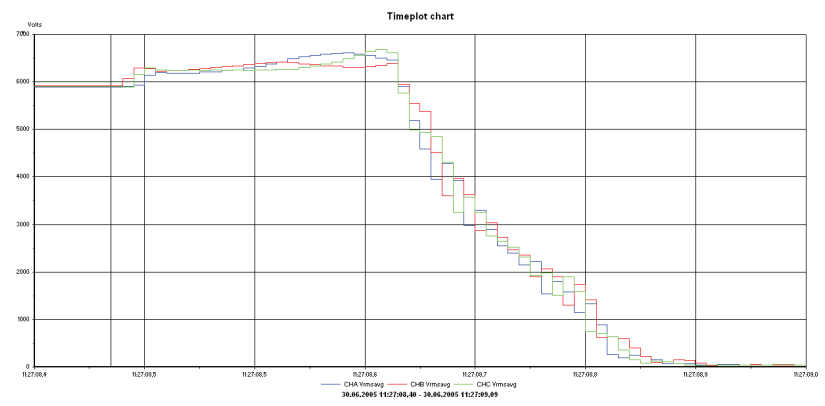

Fig. 13 Phase voltages on PCC (M1) during island operating mode on 2005-06-30

\section{Conclusion}

Power quality measurement results at the point where STPP Jakusevec is connected to the distribution network have given new knowledge on interaction of this generation utility and local distribution network.

Changes in produced energy flow can have significant effect on voltages if short circuit power at PCC is relatively small. Conducted measurements on the other hand have shown that operation of STPP Jakusevec does not influence voltages at PCC nor flicker severity, that is to say that voltage fluctuations and flickers are in no correlation with operation of generation utility.

Operating synchronous generators in distribution network can cause significant increase in generation of current harmonics, because voltage distortion is more expressed in distribution networks, then in standard generation utilities that are connected to the transmission network. Existence of current harmonics in generators can be explained by the fact that current harmonics existing in the network, that are caused by nonlinear loads, close through the generator's windings due to generator's impedance. But voltage and current harmonics measurements have shown that operation of this utility has no negative effect on voltage harmonic distortion at PCC.

Measurement results show that all voltage parameters at PCC for STPP Jakusevec are below limits determined by standard EN 50160.

Measurements made during switching operations have shown that island operation mode isn't possible because of network configuration and generation utility characteristics.

Based on conducted measurements it can be said that operation of small thermal power plant Jakusevec has no significant effect on power quality at point of common coupling and that all conditions for safe and reliable operation of this generation utility parallel with distribution network are established.

\section{References}

[1] EN 50160: 1999, Voltage characteristics of electricity supplied by public distribution systems.

[2] IEC/TR 61000-3-6: 1996, Electromagnetic compatibility (EMC)- Part 3:Limits - Section 6: Assessment of emission limits for distorting loads in MV and HV power systems - Basic EMC publication.

[3] IEC/TR 61000-3-7: 1996. Electromagnetic compatibility (EMC)- Part 3: Limits - Section 7: Assessment of emission limits for fluctuating loads in $\mathrm{MV}$ and $\mathrm{HV}$ power system - basic EMC publication. 